\title{
Neurabin: a key factor in the specific neuroprotection mediated by Adenosine
}

\author{
Presented by: Maria P. Abbracchio
}

\author{
Flaminio Cattabeni
}

Published online: 20 September 2012

(C) Springer Science+Business Media B.V. 2012

Yunjia Chen, Yin Liu, Christopher Cottingham, Lori McMahon, Kai Jiao, Paul Greengard and Qin Wang (2012) Neurabin Scaffolding of Adenosine Receptor and RGS4 Regulates Anti-seizure Effect of Endogenous Adenosine J. Neurosci. 32(8):2683-2695

\section{Summary of the article}

In this article, it is shown that the A1 Adenosine receptor (A1R) is a G-protein coupled receptor (GPCR) that is regulated by the activity of Regulator of G-protein Signaling (RGS4), which functions as a GTPase-activating protein to terminate G-protein signaling [5]. The possible role of RGS4 in adenosine-evoked signaling neuroprotection has been investigated, and it is shown that a new interacting protein, neurabin, forms a scaffold between A1R and RGS4, thus regulating the activity of A1R. It is also demonstrated that inhibiting neurabin or deleting its gene in mice provides a mean to enhance the neuroprotective effects of endogenous adenosine released during brain ischemia or during kainate induced seizures. Thus, neuroprotection can be achieved through a mechanism that does not require administration of A1R agonists, which would have important peripheral side effects. Indeed, it has already been shown that molecules inhibiting RGS4 functional activity, like CCG-4986 [4], reduce kainate-induced seizure activity in mice in vivo.

F. Cattabeni $(\square)$

Department of Pharmacological and Biomolecular Sciences Laboratory of Molecular and Cellular Pharmacology of Purinergic

Transmission, University of Milan,

Via Balzaretti 9,

20133 Milano, Italy

e-mail: fcattabeni@gmail.com

\section{Commentary}

Neurabin is specifically expressed in neural tissues with special reference to cortex, hippocampus and cerebellum [3]. This protein belongs to a family of scaffolding proteins and its homolog, spinophilin, has been shown to interact with a number of GPCRs like the Dopamine D2 [7], $\alpha 1$ and $\alpha 2$ adrenaline [9], and M2 and M3 muscarinic actylcholine [2] receptors. Neurabin has been also shown to sequester RGS2 from binding to $\alpha_{1} \mathrm{AR}$, thus enhancing $\alpha_{1} \mathrm{AR}$ mediated signaling [10]. Moreover, deleting the gene of spinophilin reduces the analgesic effects of acute morphine administration, but enhances adaptation to sub-chronic morphine exposure, including increased morphine dependence, place-conditioning and analgesic tolerance [1].

However, the direct interaction of neurabin or spinophilin with these GPCRs had never been shown before. By studying the interaction of neurabin with A1R at molecular level, this article fills this gap. The interaction neurabin-A1R occurs at the third intracellular loop of A1R and the Cterminal tail of neurabin (amino acids 146 and 453).

Neurabin interacts with A1R only when the agonist binds to its receptor and its binding attenuates receptor signaling: therefore inhibition of neurabin enhances the effect of A1R agonists, like R-PIA, and its sedative effects. It is important to note that modifications in the activity of neurabin occurs always in the absence of any modification of A1R numbers or affinity.

It has always been suggested that adenosine could serve as an important neuroprotectant and anti-seizure compound $[6,8]$ via A1Rs, but its therapeutic efficacy has always been questioned by the potential side effects of A1 agonists at different peripheric organs and systems. By showing that adenosine induced neuroprotection can be achieved by a 
direct interaction with neurabin that does not require administration of adenosine A1R agonists, this article opens up the possibility to exploit the adenosinergic system to obtain molecules able to show therapeutic efficacy specifically at the level of CNS.

\section{Future directions of research}

It will be interesting to find novel compounds able to suppress neurabin functional activity. By knowing neurabin gene structure, it should be possible to design new molecular entities able to disrupt or prevent the formation of the A1R/neurabin/RGS4 complex. It should be pointed out, once again, that this should result in a specific effect at the level of CNS and therefore make possible to exploit the adenosinergic system for selective therapeutic interventions in the field of neurodegenerative diseases, an area in a tremendous need of new and effective drugs.

\section{References}

1. Charlton JJ, Allen PB, Psifogeorgou K, Chakravarty S, Gomes I, Neve RL, Devi LA, Greengard P, Nestler EJ, Zachariou V (2008) Multiple actions of spinophilin regulate mu opioid receptor function. Neuron 58:238-247

2. Kurogi M, Nagatomo K, Kubo Y, Saitoh O (2009) Effects of spinophilin on the function of RGS8 regulating signals from M2 and M3-mAChRs. Neuroreport 20:1134-1139

3. Nakanishi H, Obaishi H, Satoh A, Wada M, Mandai K, Satoh K, Nishioka H, Matsuura Y, Mizoguchi A, Takai Y (1997) Neurabin: a novel neural tissue-specific actin filament-binding protein involved in neurite formation. J Cell Biol 139:951-961
4. Roman DL, Blazer LL, Monroy CA, Neubig RR (2010) Allosteric inhibition of the regulator of $\mathrm{G}$ protein signalingGalpha protein-protein interaction by CCG-4986. Mol Pharmacol 78:360-365

5. Ross EM, Wilkie TM (2000) GTPase-activating proteins for heterotrimeric $\mathrm{G}$ proteins: regulators of $\mathrm{G}$ protein signaling (RGS) and RGS-like proteins. Annu Rev Biochem 69:795-827

6. Sebastiao AM, Ribeiro JA (2009) Adenosine receptors and the central nervous system. Handb Exp Pharmacol 193:471-534

7. Smith FD, Oxford GS, Milgram SL (1999) Association of the D2 dopamine receptor third cytoplasmic loop with spinophilin, a protein phosphatase-1-interacting protein. J Biol Chem 274:1989419900

8. Stone TW (2002) Purines and neuroprotection. Adv Exp Med Biol 513:249-280

9. Wang Q, Zhao J, Brady AE, Feng J, Allen PB, Lefkowitz RJ, Greengard P, Limbird LE (2004) Spinophilin blocks arrestin actions in vitro and in vivo at $G$ protein-coupled receptors. Science 304:1940-1944

10. Wang X, Zeng W, Kim MS, Allen PB, Greengard P, Muallem S (2007) Spinophilin/neurabin reciprocally regulate signaling intensity by $\mathrm{G}$ proteincoupled receptors. EMBO J 26:2768-2776

\section{About the author}

Flaminio Cattabeni, Ph.D. in Biochemistry (1968) at the University of Milano, Italy. Post-Doctoral Fellow at the National Institute of Mental Health, USA. From 1984 till 2011, Full Professor of Pharmacology, School of Pharmacy, University of Milano and former Head of the Ph.D. Programme on Toxicology, University of Milano.

Former President of the Federation of the European Pharmacological Societies (EPHAR) and co-Founder of the Purine Club and its Past President.

Author of more than 239 articles published in internationally refereed journals and 78 Chapters in Books or Proceedings of International Meetings. 